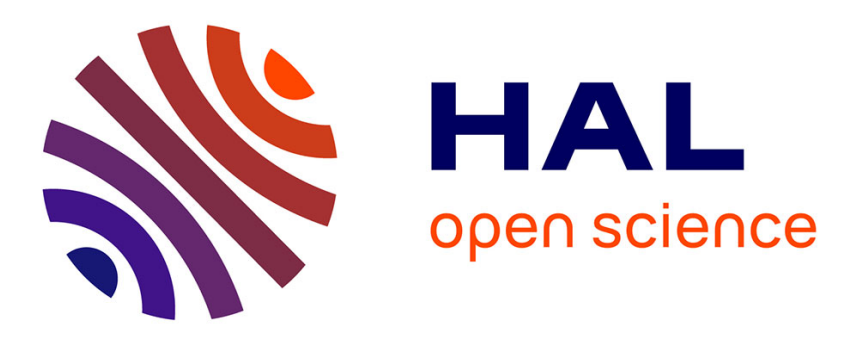

\title{
Amélioration de la reconnaissance et de l'imitation d'expressions faciales chez des enfants autistes grâce à une présentation visuelle et sonore ralentie
} France Lainé, Carole Tardif, Bruno Gepner

\section{- To cite this version:}

France Lainé, Carole Tardif, Bruno Gepner. Amélioration de la reconnaissance et de l'imitation d'expressions faciales chez des enfants autistes grâce à une présentation visuelle et sonore ralentie. Annales Médico-Psychologiques, Revue Psychiatrique, 2008, 166, pp.533-538. hal-01787281

\author{
HAL Id: hal-01787281 \\ https://hal.science/hal-01787281
}

Submitted on 7 May 2018

HAL is a multi-disciplinary open access archive for the deposit and dissemination of scientific research documents, whether they are published or not. The documents may come from teaching and research institutions in France or abroad, or from public or private research centers.
L'archive ouverte pluridisciplinaire HAL, est destinée au dépôt et à la diffusion de documents scientifiques de niveau recherche, publiés ou non, émanant des établissements d'enseignement et de recherche français ou étrangers, des laboratoires publics ou privés. 


\title{
Page de titre
}

\section{Amélioration de la reconnaissance et de l'imitation d'expressions faciales chez des enfants autistes grâce à une présentation visuelle et sonore ralentie}

\author{
France Lainé ${ }^{1}$, Carole Tardif ${ }^{1}$, Bruno Gepner ${ }^{2}$
}

${ }^{1}$ : PsyCLE, Centre de Psychologie de la Connaissance, du Langage et de l'Emotion, EA 3273, Université de Provence, UFR de Psychologie, 29 av. R. Schuman, 13621 Aix en Provence Cedex 1. Tél : 0442933990

${ }^{2}$ : Centre Hospitalier Montperrin et Laboratoire Parole et Langage, UMR CNRS 6057, Aix-en-Provence

E-mail de 1'auteur : france_laine@hotmail.com

Titre abrégé : Expressions faciales ralenties et autisme 


\section{Résumés et mots-clés}

\section{Résumé français :}

De nombreuses études ont montré que les personnes autistes souffrent de troubles perceptifs visuels et auditifs, pouvant affecter leur compréhension de la communication. Récemment, des travaux ont montré que les personnes autistes ont des difficultés à percevoir les mouvements du visage impliqués dans la communication ainsi que les sons de la parole car ceux-ci seraient trop rapides pour elles. Nous avons cherché à savoir dans cette étude si un ralentissement de la voix humaine et des mouvements faciaux entraînerait une amélioration de la reconnaissance d'expressions faciales présentées de manière dynamique chez des enfants autistes comparés à des enfants normaux. Nos résultats montrent que le ralentissement d'expressions faciales et de leur son correspondant en améliore la reconnaissance et induit davantage d'imitations chez les enfants autistes par rapport aux enfants témoins. Un logiciel permettant de ralentir le son et l'image de manière synchrone pourrait alors aider les personnes autistes à mieux comprendre les messages verbaux et non verbaux lors des échanges interpersonnels.

\section{English summary:}

An increasing amount of studies demonstrated the existence of visual physical and biological motion integration deficit in individuals with autism, especially when movement is rapid. Since visual motion is intrinsically involved in social interactions through movements of the body and face, visual motion processing deficit could therefore account for their verbal and non verbal comprehension and social interactions disorders. Besides, other studies 
revealed the existence of speech integration deficit in this population, which could also account for their verbal comprehension deficits. The aim of our study is to test whether slowing down sound flow and facial movement enhance facial recognition and imitation by autistic children compared to normal control children. Results show that slow dynamic presentation enhances recognition and imitation of facial expressions by autistic children. Our perspective is to elaborate a software, that could simultaneously slow down facial movements and speech flow, and to assess its impact on the comprehension of words and instructions by autistic children.

Mots-clés : autisme ; communication ; expressions faciales émotionnelles et non émotionnelles ; perception du mouvement et des sons ; ralentissement.

Keywords : autism; communication; emotional and non emotional facial expressions ; motion and sound perception ; slowing down.

\section{Texte}

\section{Introduction}

L'autisme se caractérise par des troubles de la communication verbale et non verbale et des interactions sociales [2], étudiés par de nombreux chercheurs, tant au niveau de l'expression que de la compréhension. Hobson (1991) a notamment montré que les personnes autistes ont des difficultés à reconnaître les émotions faciales impliquées dans les interactions sociales. Notons qu'une émotion faciale implique des mouvements du visage et que sa reconnaissance est ainsi en partie liée au traitement de la dynamique faciale [37]. Le mouvement semble donc être nécessaire à la reconnaissance et à la 
compréhension des émotions. Ainsi, on peut se demander si les personnes autistes ont une difficulté à traiter les expressions faciales émotionnelles en soi, comme le suggère Hobson (1991), ou si elles ont une difficulté plus générale à traiter la mobilité faciale de ces expressions. Certaines études, en faveur de la seconde hypothèse, ont montré chez des personnes autistes d'une part que le traitement des aspects dynamiques du visage (lecture du regard et lecture labiale) est déficitaire $[3,5,15,17,27]$, et d'autre part l'existence d'un trouble perceptif et/ou intégratif de la vision des mouvements physiques et biologiques [4, 20, 30, 40, pour une revue, voir 29]. Il semblerait aussi que plus les mouvements sont rapides, plus les personnes autistes ont du mal à les percevoir et à les intégrer $[18,19,28$, pour une revue, voir 21]. En regard de ces données, et comme le suggère une étude pilote [16], Gepner $(2001,2004)$ suppose qu'un ralentissement de ces mouvements faciaux permettrait aux personnes autistes de mieux percevoir et reconnaître les expressions faciales émotionnelles et non émotionnelles.

Ces différentes études montrent les difficultés des personnes autistes au niveau de la communication non verbale, et leur lien possible avec un trouble de la perception visuelle du mouvement. Or, ce niveau n'est pas le seul touché dans l'autisme. Le domaine de la communication verbale l'est également. En effet, les personnes autistes semblent ne pas intégrer correctement des sons non verbaux $[1,31,32]$ mais également, d'après une étude récente, ne pas percevoir les sons de la parole [22]. Tardif et al. (2002) ont quant à eux montré chez des enfants autistes que la catégorisation de certains phonèmes présentés en vitesse normale était déficitaire, et que leur ralentissement permettait aux enfants autistes de mieux les percevoir et les catégoriser. 
Ainsi, de manière générale, il semblerait que la parole et les mouvements faciaux aillent trop vite pour être perçus correctement par les personnes autistes, ceci pouvant les gêner dans le traitement de certains aspects de la communication verbale et non verbale. Un ralentissement de la parole et des mouvements faciaux pourrait donc être bénéfique à leur compréhension des échanges interpersonnels. Dans la présente étude, nous avons voulu tester l'hypothèse selon laquelle une présentation ralentie d'expressions faciales et de leur son correspondant améliore leur reconnaissance et induit davantage leur imitation chez des enfants autistes, comme l'ont suggéré Gepner et al. (2001).

\section{Matériel et méthode}

Pour tester notre hypothèse, nous avons proposé une tâche de reconnaissance d'expressions faciales émotionnelles et non émotionnelles dynamiques dans différentes conditions de son et de vitesse, à un groupe de 12 enfants autistes, et à deux groupes témoins de 12 enfants chacun, appariés individuellement aux enfants autistes sur la base du sexe et de l'âge mental verbal (mesuré à l'aide du TVAP : Test de Vocabulaire Actif/Passif, [10]) pour un groupe et sur la base du sexe et de l'âge mental non verbal (visuo-spatial) (mesuré à l'aide des matrices de Raven, [36]) pour l'autre groupe.

\section{Participants}

Les enfants autistes recrutés ont été diagnostiqués selon les critères du DSM IV [2] et le degré de leur atteinte évalué à l'aide de la CARS (Childhood Autism Rating Scale : Echelle d'évaluation de l'autisme infantile) [38] afin de constituer deux sous-groupes: l'un avec un autisme léger $(\mathrm{CARS}<35)$ et l'autre avec un autisme modéré à sévère (CARS>=35). 
Les 12 enfants autistes ont un âge réel compris entre 7 ans 4 mois et 14 ans 4 mois (moyenne $=10 ; 8 /$ écart-type $=2 ; 6$ ). Leur âge mental verbal est compris entre 3 ans et 7 ans 9 mois $($ moyenne $=5 ; 2$ / écart-type $=1 ; 4)$ et leur âge mental non verbal est compris entre 3 ans et 11 ans (moyenne $=6 ; 11 /$ écart-type $=2 ; 6)$. Ces enfants ont été recrutés dans des établissements médicosociaux et scolaires spécialisés des Bouches-du-Rhône.

Le premier groupe d'enfants témoins, appariés aux enfants autistes sur le sexe et l'âge mental verbal, est composé de 12 enfants ordinaires, d'âge compris entre 3 ans 3 mois et 7 ans 1 mois (moyenne $=5 ; 1 /$ écart-type $=1 ; 4$ ). Le second groupe d'enfants témoins, appariés aux enfants autistes sur le sexe et l'âge mental non verbal, est composé de 12 enfants ordinaires d'âge compris entre 3 ans 4 mois et 11 ans 1 mois (moyenne $=6 ; 1 /$ écart-type $=2 ; 6$ ). Les enfants témoins ont été recrutés dans des écoles maternelles et primaires ordinaires des Bouches-du-Rhône.

\section{Protocole expérimental}

Présentée sur ordinateur, la tâche expérimentale consiste à reconnaître quatre expressions émotionnelles (joie, tristesse, dégoût, surprise) et quatre expressions non émotionnelles (prononciation des voyelles $\mathrm{A}, \mathrm{O}, \mathrm{I}$, protrusion de la langue) produites par une actrice professionnelle. Chaque expression faciale est présentée sur 2 secondes et selon quatre conditions de vitesse ainsi nommées :

-V0 : vitesse nulle, i.e. présentation contrôle statique de l'expression.

-V lente: vitesse de référence produite par l'actrice et considérée comme lente $[16,33,36]$

$-2 \mathrm{x} V$ lente : ralentissement de $\mathrm{V}$ lente par deux (vitesse très lente) 
- 1/2 V lente : accélération de $\mathrm{V}$ lente par deux (vitesse rapide de la vie quotidienne)

Enfin, chaque expression faciale est présentée soit de manière muette, soit accompagnée du son correspondant (par exemple « Ouais !» pour la joie). Le son est synchronisé à la vitesse de présentation de l'expression faciale.

L'expérience comprend au total 64 séquences, les 8 expressions faciales étant présentées dans toutes les modalités de passation dans un ordre aléatoire ((4 Emotions +4 Non-émotions) $\times 2$ Son/Non-son x 4 Vitesses $).$

\section{Procédure expérimentale}

L'enfant est assis face à l'écran d'un ordinateur portable, situé à $30 \mathrm{~cm}$ de ses yeux et à sa hauteur. Durant toute la passation (filmée pour analyses ultérieures), une expérimentatrice, assise à ses côtés, note ses réponses et l'incite à concentrer son attention sur l'écran. Une autre expérimentatrice, face à lui, note les imitations faciales et/ou vocales qu'il produit en fonction de l'expression faciale visualisée.

Chaque séquence est suivie d'un intervalle d'une seconde où l'écran est noir, puis 4 photos apparaissent, deux en haut et deux en bas de l'écran, dans un ordre aléatoire. Parmi ces 4 photos, l'enfant doit, sans limitation de temps, retrouver l'expression cible qu'il vient de voir et la montrer en la pointant.

Huit séquences de démonstration ont été extraites des 64 séquences, représentant un exemple de chacune des expressions faciales et des modalités de présentation visuelle et sonore. Elles permettent de donner à l'enfant la consigne à l'aide d'exemples concrets.

Deux mesures sont effectuées à partir des réponses des enfants : 
1) mesure du score de chaque enfant en reconnaissance des expressions faciales : les réponses correctes sont cotées 1 et les réponses incorrectes 0 .

2) mesure du nombre d'imitations faciales et/ou vocales induites chez chaque enfant par la présentation des expressions faciales : seules les imitations faciales et/ou vocales correspondant à l'expression cible sont cotées.

\section{Résultats}

Nous avons procédé à des analyses de variance (ANOVA) sur les résultats en reconnaissance et en imitation, sur les trois groupes d'enfants, selon le schéma suivant : 3 (Groupe) x 2 (Type d'expression faciale) x 4 (Vitesse de présentation) $\mathrm{x} 2$ (Condition sonore), avec le score en reconnaissance d'expressions faciales et le nombre d'imitations comme variables dépendantes et le type d'expression faciale, la vitesse de présentation et la condition sonore comme mesures répétées.

\section{$\left.1^{\circ}\right)$ Scores en reconnaissance des expressions faciales}

\section{a) Comparaisons inter-groupes}

Les enfants autistes reconnaissent significativement moins d'expressions faciales que les enfants témoins appariés sur l'âge mental verbal et sur l'âge mental non verbal $[\mathrm{F}(2,33)=10.58$ et $\mathrm{p}<.0003]$, quels que soient le type d'expression et les conditions de son et de vitesse, alors qu'aucune différence significative n'est retrouvée entre les deux groupes témoins (voir figure 1).

Les analyses suivantes ne concernent que les enfants autistes, les différences significatives observées dans ce groupe n'étant pas présentes dans les groupes témoins.

\section{b) Comparaisons intra-groupe dans le groupe des enfants autistes}


Concernant le facteur «Type d'expression», les enfants autistes reconnaissent significativement moins d'expressions faciales émotionnelles que d'expressions faciales non émotionnelles $[F(1,11)=16.09$ et $\mathrm{p}<.0003$ ].

Concernant le facteur «Son», les enfants autistes reconnaissent significativement plus d'expressions faciales sonorisées que muettes $[\mathrm{F}(1$, $11)=10.43$ et $p<.008]$. Une comparaison planifiée révèle que cette différence n'est valable que dans la condition statique V0 $[F(1,33)=19,37 ; \mathrm{p}<.0001]$.

Concernant le facteur «Vitesse », les enfants autistes reconnaissent significativement plus d'expressions faciales présentées en $\mathrm{V}$ lente que dans les trois autres conditions de vitesse (analyses post-hoc à $\mathrm{p}<.02$ ), et aucune différence significative n'est retrouvée entre ces trois dernières conditions de vitesse (V0, $2 \times \mathrm{V}$ lente, $1 / 2 \mathrm{~V}$ lente) (voir figure 2). De plus, un test exact de Fischer montre que les enfants atteints d'autisme modéré à sévère sont significativement plus aidés par les vitesses $\mathrm{V}$ lente et $2 \times \mathrm{V}$ lente $(\mathrm{p}<.05)$ pour reconnaître les expressions faciales, comparativement aux enfants atteints d'autisme léger dont les scores ne varient pas en fonction de la vitesse de présentation (voir tableau I).

\section{$2^{\circ}$ ) Scores en imitation des expressions faciales}

Les enfants autistes imitent significativement plus les expressions présentées que les enfants témoins $[\mathrm{F}(2,33)=9.40$ et $\mathrm{p}<.0005]$ (voir figure 3). Ils imitent davantage les vocalisations (moyenne $=13,33$; écart-type $=12,28$ ) que les mimiques faciales (moyenne $=6,25$; écart-type $=8,4)[\mathrm{F}(1,21)=7.34$ et $\mathrm{p}<.01]$. Ainsi, les items sonorisés sont davantage imités que les items non sonorisés $[F(1,11)=13.27$ et $p<.003]$. De plus, ces imitations sont significativement plus induites lors des présentations lente et très lente : les 
imitations faciales sont davantage induites en $2 \times \mathrm{V}$ lente qu'en $\mathrm{V}$ lente $[\mathrm{F}(1$, $11)=4.84$ et $p<.05]$, les imitations vocales sont davantage induites en $\mathrm{V}$ lente qu'en $1 / 2 \mathrm{~V}$ lente $[\mathrm{F}(1,11)=5.85$ et $\mathrm{p}<.03]$.

L'induction d'imitations chez les enfants autistes n'entraîne pas forcément une meilleure reconnaissance des expressions imitées.

\section{Discussion}

Les principaux résultats de notre étude sont les suivants :

1) les enfants autistes reconnaissent moins d'expressions faciales émotionnelles et non émotionnelles que les deux groupes d'enfants témoins.

2) la présentation en vitesse lente révèle le meilleur score de reconnaissance chez les enfants autistes.

3) les enfants ayant les degrés les plus élevés d'autisme sont les plus aidés par les vitesses lente et très lente dans leur reconnaissance des expressions faciales.

4) les enfants autistes reconnaissent moins les expressions émotionnelles que les expressions non émotionnelles

5) les enfants autistes reconnaissent moins les expressions muettes que les expressions sonorisées.

6) les enfants autistes imitent plus que les enfants témoins et davantage les vocalisations que les mimiques faciales. Ces imitations sont davantage induites par les vitesses lente et très lente.

Notre premier résultat va dans le sens de nombreux travaux antérieurs effectués depuis près de 20 ans [6, 7, 24, 33], et récemment confirmés par des études en IRMf [8, 39]: les enfants autistes reconnaissent moins les 
expressions faciales que les enfants témoins appariés sur l'âge mental verbal et non verbal. Leur difficulté de reconnaissance des expressions faciales ne peut donc pas être expliquée seulement par un retard de développement. Nous supposons que l'existence d'un trouble de la perception des mouvements, notamment des mouvements rapides, pourrait expliquer cette difficulté. En effet, notre second résultat va dans ce sens et confirme d'autres études $[13,16$, 19]. De plus, d'après le troisième résultat, ce bénéfice apporté par le ralentissement des expressions faciales (en vitesses lente et très lente) est observé uniquement chez les enfants dont le degré d'autisme est modéré à sévère : plus les enfants ont un degré d'autisme important, plus ils semblent bénéficier du ralentissement des expressions pour les reconnaître donc plus leur atteinte perceptive visuelle du mouvement est importante $[4,18]$. Ce résultat va dans le sens d'une corrélation entre degré de sévérité d'autisme et degré d'altération perceptivo-cognitive [2].

Concernant notre quatrième résultat, il peut également être expliqué par ce trouble perceptif; en première analyse, il tendrait à confirmer la thèse de Hobson (1991) supposant que les enfants autistes ont un trouble spécifique de la reconnaissance des signaux émotionnels véhiculés notamment par les expressions faciales et les indices vocaux. Cependant, d'autres auteurs récusent cette spécificité d'un désordre de traitement des signaux émotionnels en tant que tel [9]. En effet, notre étude et d'autres travaux antérieurs de notre équipe $[15,17]$ ont montré que les enfants autistes reconnaissent également moins d'expressions non émotionnelles que les enfants des groupes témoins. Il n’y aurait donc pas uniquement un désordre du traitement des signaux émotionnels. Toutefois, au sein du groupe des enfants autistes, les expressions émotionnelles 
sont moins reconnues que les expressions non émotionnelles. Plusieurs études ont mis en évidence que les enfants autistes privilégiaient l'analyse locale (portant sur un élément du visage comme les lèvres ou les yeux), à l'analyse configurale (portant sur les relations spatiales entre les éléments du visage et mise en jeu dans la perception des mouvements faciaux) [11, 41, 43]. Or, une expression émotionnelle mobilise davantage de mouvements faciaux qu'une expression non émotionnelle [34] et sollicite donc davantage une analyse configurale pour être identifiée. Des observations ont également révélé la tendance qu'ont les enfants autistes à éviter du regard le mouvement des yeux de l'interlocuteur [26], utile dans l'analyse d'une émotion mais considéré comme trop rapide [23] et rendant ainsi difficile l'analyse configurale. De plus, dans notre étude, cette différence de reconnaissance entre expressions émotionnelles et non émotionnelles est atténuée en présentation lente et très lente, ce qui pourrait signifier que le ralentissement des mouvements faciaux faciliterait l'analyse configurale de l'expression en laissant le temps à l'enfant autiste de traiter davantage d'informations pertinentes $[14,16]$, notamment au niveau de la région des yeux. Ce quatrième résultat peut donc aussi être expliqué par un défaut de vision des mouvements.

Notre cinquième résultat montre que l'adjonction du son correspondant à l'expression faciale présentée en condition statique, dans laquelle le son est présenté lentement et naturellement, en améliore la reconnaissance chez les enfants autistes. On peut penser que l'impact du son est d'autant plus net que l'information visuelle fournie par l'expression faciale est moins informative. A l'opposé, quand l'expression faciale est dynamique, elle renseigne suffisamment l'enfant autiste et l'adjonction d'un son ne rajoute rien. 
Le dernier résultat montre que les imitations sont davantage induites lorsque les expressions faciales sont sonorisées et qu'elles sont plutôt vocales que faciales. Ainsi, le son semble jouer un rôle important dans l'induction des imitations chez les enfants autistes. D'autre part, il est intéressant de noter que ces imitations sont davantage induites dans les vitesses ralenties, comme l'avaient noté Gepner et al. (2001), sans toutefois entraîner systématiquement une bonne reconnaissance de l'expression. Ceci signifie que lorsque les mouvements faciaux et les vocalisations engagées dans l'expression faciale sont présentés de manière ralentie, l'enfant est plus à même de les percevoir.

Sur le plan neurofonctionnel, les difficultés des enfants autistes dans le traitement des mouvements faciaux sont probablement en partie liées à des dysfonctionnements au niveau du sillon temporal supérieur. Cette région cérébrale est engagée dans le traitement perceptif des mouvements biologiques tels que les mouvements des mains, du corps, de la bouche (dont la lecture labiale) et des yeux [pour une revue, voir 35] mais également dans le traitement de la voix humaine [22]. Une anomalie de fonctionnement de cette région pourrait donc être impliquée dans les anomalies de perception des mouvements faciaux et du flux sonore chez les personnes autistes.

En conclusion, les résultats de notre étude tendent à confirmer l'hypothèse de Gepner (2001, 2004) selon laquelle le ralentissement des mouvements faciaux permettrait une meilleure reconnaissance des expressions faciales. Ils montrent aussi que le ralentissement des sons vocaux permet une meilleure reconnaissance des mimiques faciales et induit leur imitation.

Notre étude, suite aux travaux antérieurs de notre équipe, est la première à démontrer l'utilité du ralentissement des expressions faciales et du 
son correspondant pour favoriser leur reconnaissance et leur imitation par des enfants autistes. Dès lors, une des perspectives de poursuite de notre travail est de créer un logiciel informatique permettant d'améliorer la compréhension des interactions sociales et communicatives par les personnes autistes en ralentissant les mouvements faciaux, la parole et les vocalisations de leur interlocuteur, de manière synchrone et automatique.

\section{Références bibliographiques}

1 Adrien J-L. Autisme du jeune enfant. Paris: Expansion scientifique française; 1986.

2 American Psychiatric Association. Diagnostic and Statistical Manual of Mental Disorders (DSM IV). Washington, D.C.: American Psychiatric Association; 1994.

3 Baron-Cohen S, Campbell R, Karmiloff-Smith A, Grant J, Walker J. Are children with autism blind to the mentalistic significance of the eyes? Br J Dev Psychol. 1995;13:379-98.

4 Blake R, Turner LM, Smoski MJ, Pozdol SL, Stone WL. Visual Recognition of Biological Motion is Impaired in Children with Autism. Psychol Sci. $2003 ; 14: 151-7$.

5 Bormann-Kischkel C, Vilsmeier M, Baude B. The development of emotional concepts in autism. J Child Psychol Psychiatry. 1995;36:1243-59.

6 Boucher J, Lewis V. Unfamiliar face recognition in relatively able autistic children. J Child Psychol Psychiatry. 1992;33:843-59.

7 Braverman M, Fein D, Lucci D, Waterhouse L. Affect comprehension in children with pervasive developmental disorders. J Autism Dev Disord. 1989;19:301-16. 
8 Critchley HD, Daly EM, Bullmore ET, Williams SCR, Van Amelsvoort T, Robertson DM, et al. The functional neuroanatomy of social behaviour: Changes in cerebral blood flow when people with autistic disorder process facial expressions. Brain. 2000;123:2203-12.

9 Davies S, Bishop D, Manstead ASR, Tantam D. Face Perception in Children with Autism and Asperger's Syndrome. J Child Psychol Psychiatry. $1994 ; 35: 1033-57$.

10 Deltour JJ, Hupkens D. Test de Vocabulaire Actif Passif, TVAP. Paris: Éditions EAP; 1980.

11 Deruelle C, Rondan C, Fagot J, Gepner B. Local, global and configural processing of faces in autistic children. Int J Psychol. In press 2005.

12 Frith U. Autism : explaining the enigma. Oxford : Basic Blackwell, 1989.

13 Gepner B. Malvoyance du mouvement dans l'autisme infantile? Une nouvelle approche neuropsychopathologique développementale. Psychiatr Enfant. 2001;1:77-126.

14 Gepner B. Autism, movement and facial processing [letter]. Am J Psychiatry. 2004;161:1719.

15 Gepner B, Buttin C, de Schonen S. Face processing in young autistic children. Infant Behav Dev. 1994;17:661.

16 Gepner B, Deruelle C, Grynfeltt S. Motion and emotion: a novel approach to the study of face processing by young autistic children. J Autism Dev Disord. 2001;31:37-45.

17 Gepner B, de Gelder B, de Schonen S. Face processing in autistics: a generalised deficit? Child Neuropsychol. 1996;2:123-39. 
18 Gepner B, Mestre D. Postural reactivity to fast visual motion differentiates autistic from children with Asperger syndrome. J Autism Dev Disord. 2002a;32:231-8.

19 Gepner B, Mestre D. Rapid visual-motion integration deficit in autism. Trends Cogn Sci. 2002b;6:455.

20 Gepner B., Mestre D., Masson, G. \& de Schonen S. Postural effects of motion vision in young autistic children. NeuroReport. 1995;6:1211-14.

21 Gepner B., Lainé F., Tardif C. Emotion Mis-sight and Other Temporal Processing Disorders in Autism. Cah Psychol Cogn/Curr Psychol Cogn. 2005;23:104-21.

22 Gervais H, Belin P, Boddaert N, Leboyer M, Coez A, Sfaello I, et al. Abnormal cortical voice processing in autism. Nat Neurosci. 2004;7:801-2.

23 Grandin T. Penser en images. Paris: Odile Jacob; 1997.

24 Hobson RP. The autistic child's apraisal of expressions of emotion. J Child Psychol Psychiatry. 1986;27:321-42.

25 Hobson RP. What is autism ? Psychiatr Clin North Am. 1991;14:1-17.

26 Kanner L. Autistic disturbances of affective contact. Nerv Child. 1943;2:217-50.

27 Leekam S, Baron-Cohen S, Perrett D, Milders M, Brown S. (1993) . Eye direction: A dissociation between geometric and joint-attention skills in autism. Unpublished manuscript, Institute of Social Psychology, University of Kent.

28 Mestre D, Rondan C, Masson G, Castet E, Deruelle C, Gepner B. Evaluation de la vision du mouvement chez des enfants autistes au moyen du nystagmus opto-cinétique. Rev Travaux Interdisciplinaires Laboratoire Parole et Langage. 2002;21:192-8. 
29 Milne E, Swettenham J, Campbell R. Motion perception and autistic spectrum disorder: A review. Cah Psychol Cogn/Curr Psychol Cogn. $2005 ; 23: 3-36$.

30 Milne E, Swettenham J, Hansen P, Campbell R, Jeffries H, Plaisted K. High motion coherence thresholds in children with autism. J Child Psychol Psychiatry. 2002;43:255-63.

31 Mottron L, Morasse K, Belleville S. A study of Memory Functioning in Individuals with Autism. J Child Psychol Psychiary. 2001;42:253-60.

32 Mottron L, Peretz I, Ménard E. Local and Global Processing of Music in High-functioning Persons with Autism : Beyond Central Coherence ? J Child Psychol Psychiatry. 2000;41:1057-65.

33 Ozonoff S, Pennington BF, Rogers SJ. Are there emotion perception deficits in young autistic children? J Child Psychol Psychiatry. 1990;31:343-61.

34 Pollick FE, Hill H, Calder A, Paterson H. Recognising facial expression from spatially and temporally modified movements. Perception. 2003;32:81326.

35 Puce A, Perrett DI. Electrophysiology and brain imaging of biological motion. Philos Trans R Soc Lond B Biol Sci. 2003;358:435-45.

36 Raven J. Progressive Matrices. Issy-les-Moulineaux : Editions EAP, Expansion Scientifiques et Psychologiques ; 1981.

37 Sato W, Yoshikawa S. The dynamic aspects of emotional facial expressions. Cogn Emot. 2004;18:701-10.

38 Schopler E, Reichler RJ, de Vellis RF, Daly K. Toward objective classification of childhood autism: Childhood Autism Rating Scale (CARS) . J Autism Dev Disord. 1980;10:91-103. 
39 Schultz RT, Gauthier I, Klin A, Fulbright RK, Anderson AW, Volkmar F, et al. Abnormal ventral temporal cortical activity during face discrimination among individuals with autism and Asperger syndrome. Arch Gen Psychiatry. $2000 ; 57: 331-40$.

40 Spencer J, O’Brien J, Riggs K, Braddick O, Atkinson J, Wattam-Bell J. Motion processing in autism; evidence for a dorsal stream deficiency. NeuroReport. 2000;11:2765-7.

41 Tantam D, Monaghan L, Nicholson H, Stirling J. Autistic children's ability to interpret faces: A research note. J Child Psychol Psychiatry. 1989;30:62330.

42 Tardif C, Thomas K, Gepner B, Rey V. Contribution à l'évaluation du système phonologique explicite chez des enfants autistes. Parole. 2002;21:3572.

43 Weeks SJ, Hobson RP. The salience of facial expression for autistic children. J Child Psychol Psychiatry. 1987;28:137-52. 
Tableaux et figures

Tableau I : Répartition des enfants autistes selon le degré d'atteinte autistique (autisme léger vs autisme modéré à sévère) en fonction du bénéfice apporté par les vitesses $\mathrm{V}$ lente et/ou $2 \times \mathrm{V}$ lente dans la reconnaissance des expressions faciales (aidés par V lente et/ou $2 \times \mathrm{V}$ lente $v s$ non aidés par V lente et/ou $2 \times \mathrm{V}$ lente).

\begin{tabular}{|l|c|c|}
\hline & $\begin{array}{c}\text { Aidés par V lente et/ou } \\
2 \times \text { V lente }\end{array}$ & $\begin{array}{c}\text { Non aidés par V lente } \\
\text { et/ou 2 x V lente }\end{array}$ \\
\hline Autisme léger $(\mathrm{N}=4)$ & 0 & 4 \\
\hline Autisme modéré à sévère & 6 & 2 \\
$(\mathrm{~N}=8)$ & & \\
\hline
\end{tabular}




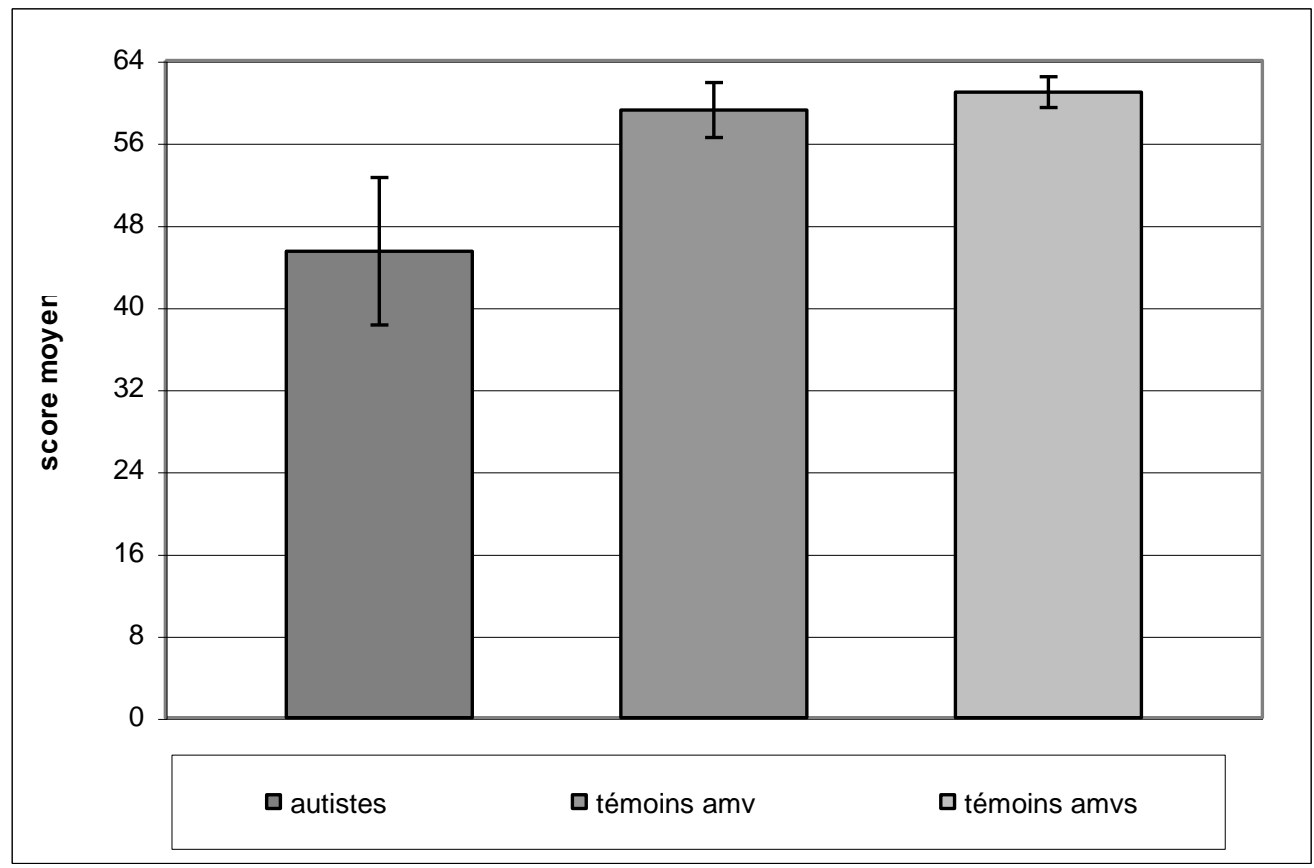

Figure 1 : Score moyen en reconnaissance des expressions faciales des enfants autistes et des enfants témoins (appariés sur l'âge mental verbal (AMV) et l'âge mental non verbal (visuo-spatial) (AMVS)).

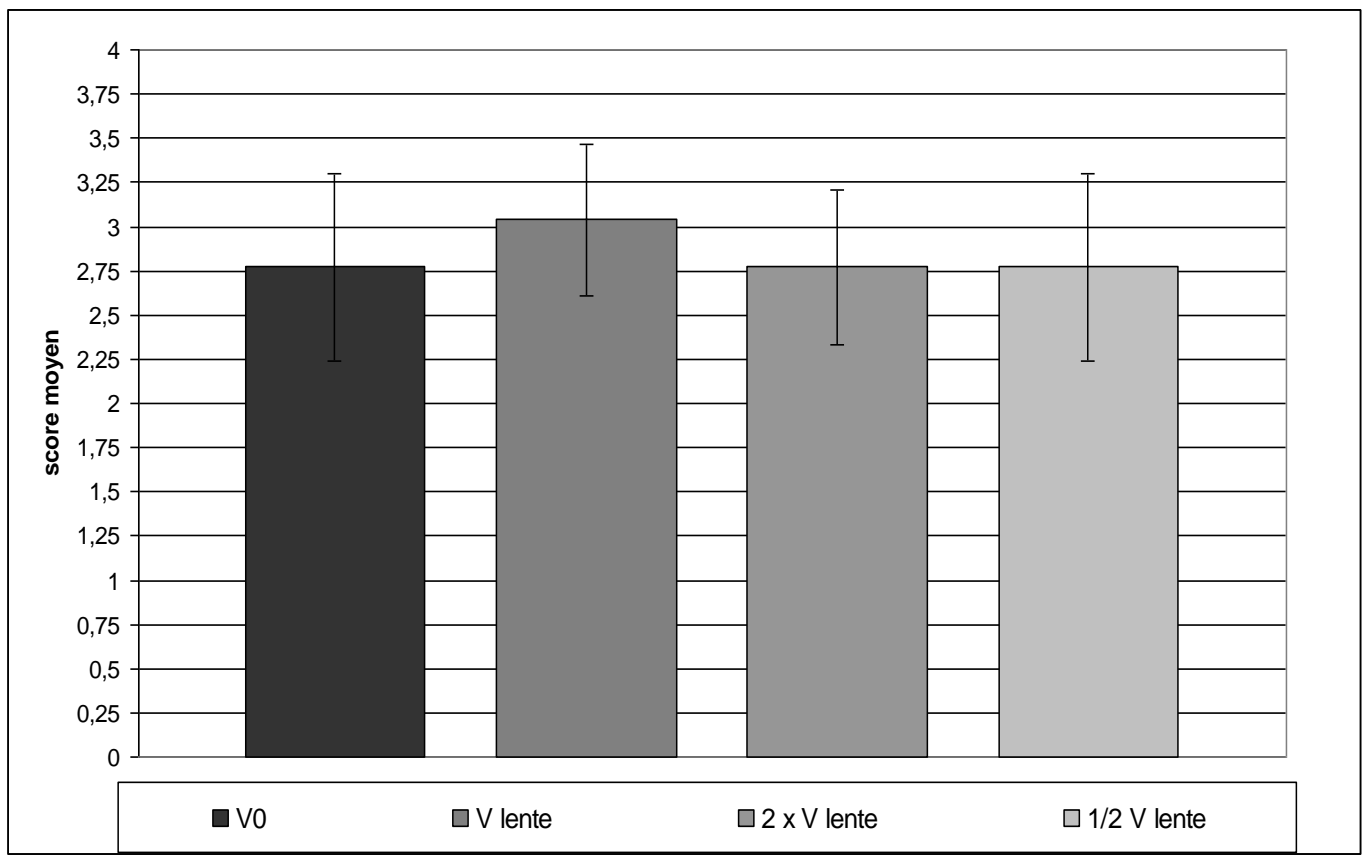

Figure 2 : Score moyen des enfants autistes en reconnaissance d'expressions faciales dans les différentes conditions de vitesse (V0, V lente, $2 \times \mathrm{V}$ lente, $1 / 2$ V lente). 


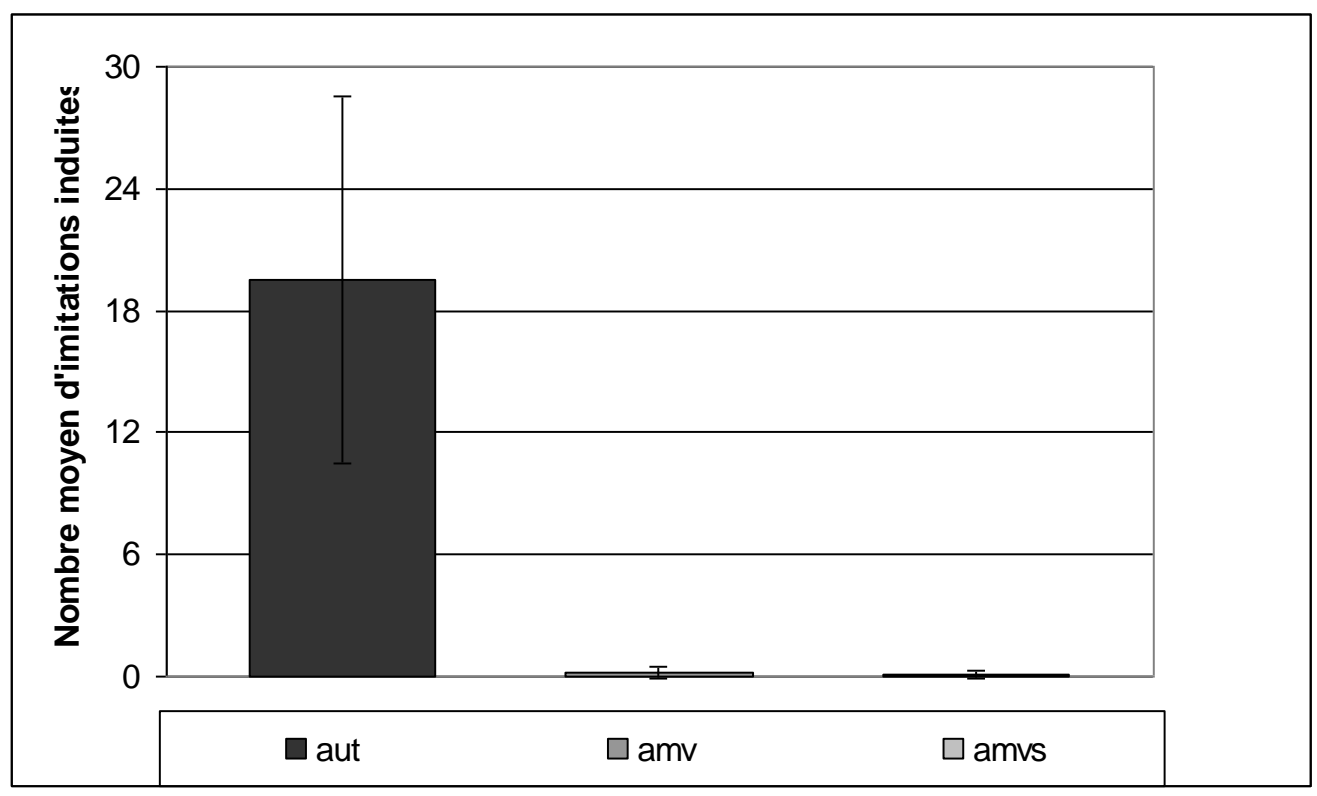

Figure 3: Nombre moyen d'imitations induites durant la passation expérimentale par groupe d'enfants (autistes, témoins AMV et témoins AMVS) ${ }^{1}$

${ }^{1}$ Les imitations produites par l'enfant sont cotées uniquement lorsqu'elles correspondent à l'expression de la séquence vue à l'ordinateur. Nous notons le nombre d'occurrences d'expressions ainsi reproduites. De ce fait, nous pouvons avoir, pour une même séquence, un nombre variable d'imitations selon l'enfant. 